\title{
$(2+1)$-dimensional wormhole from a doublet of scalar fields
}

\author{
S. Habib Mazharimousavi* and M. Halilsoy ${ }^{\dagger}$ \\ Department of Physics, Eastern Mediterranean University, Gazimağusa, Turkey
}

(Received 17 February 2015; published 24 July 2015)

\begin{abstract}
We present a class of exact solutions in the framework of $(2+1)$-dimensional Einstein gravity coupled minimally to a doublet of scalar fields. Our solution can be interpreted upon the tuning of parameters as an asymptotically flat wormhole as well as a particle model in $2+1$ dimensions.
\end{abstract}

DOI: 10.1103/PhysRevD.92.024040

PACS numbers: 04.20.Jb, 04.60.Kz

\section{INTRODUCTION}

The multiplets theory of real-valued scalar fields constitutes a model that naturally generalizes the theory of a single scalar field model [1]. The $\sigma$ model [2], the Higgs formalism [3], and the global monopole theory [4] are just a few to be mentioned in this category. Extra fields amount always to extra degrees of freedom and richness in the underlying theory. The kinetic part of the Lagrangian in this approach is proportional to $\left(\nabla \phi^{a}\right)^{2}$ (with $a$ being the symmetry group index), which is invariant under the symmetry transformations. In flat spacetime this makes a linear theory, but in a curved spacetime intrinsic nonlinearity automatically develops. The existence of an additional potential is employed as instrumental to apply spontaneous symmetry breaking in the generation of mass. Additional topological properties also are interesting subjects in this context.

Our aim in this study is, first, to add new degrees of freedom to scalar fields with internal indices in the spacetime of $(2+1)$-dimensional gravity. This amounts to considering multiplets of scalar fields and obtaining exact wormhole solutions in $2+1$ dimensions with nonzero curvature. $(2+1)$-dimensional wormholes were considered before [5]. However, such a study with scalar doublets in this particular dimension has not been conducted before. We are motivated in this line of thought mainly by the $(2+1)$-dimensional analogue of a BarriolaVilenkin-type [4] global monopole solution which is not any simpler than its $(3+1)$-dimensional counterpart [6]. We recall that the original idea of a spacetime wormhole, namely the Einstein-Rosen bridge [7], aimed to construct a geometrical model for an elementary particle. For the popularity of wormholes, however, we are indebted to the pioneering work of Morris and Thorne [8].

As expected, the invariance group in our case is $O(2)$ instead of $O(3)$. It should be added that in $2+1$ dimensions even the single scalar field solutions are very rare and restrictive [9]. This situation alone gives enough justification to search for alternatives such as the

\footnotetext{
*habib.mazhari@emu.edu.tr

†mustafa.halilsoy@emu.edu.tr
}

nonisotropic scalar multiplets. Second, we show that the solution obtained is a wormhole solution with the particular redshift function $\Phi(r)=0$, leaving us with the shape function $b(r)$. It should be emphasized that vanishing of the redshift function is not a choice but is rather imposed as a result of the field equations. Our wormhole is powered by an exotic matter [10], and the scalar field doublet $\phi^{a}(r, \theta)$ is expressed in transcendental Lambert functions. When these are brought together, our solution for the wormhole becomes supported by a phantom scalar field doublet. Wormholes with a phantom scalar in $3+1$ dimensions were studied in [11]. Phantom wormholes in $2+1$ dimensions were considered in [12]. Another interpretation for our solution can be considered à la Einstein and Rosen to represent a localized particle model in $2+1$ dimensions. We wish to comment that $(2+1)$-dimensional gravity gained enough prominence during recent decades due to the discovery of a cosmological black hole [13]. This gave birth to the general consensus among relativists that the $(2+1)$-dimensional geometrical structures such as black holes and wormholes provide useful test beds for understanding their higherdimensional cousins. Within this context, we see certain advantages in studying and understanding better the $(2+1)$ dimensional wormhole solutions.

The organization of the paper is as follows. In Sec. II we introduce our action and derive the field equations. We solve and plot the metric function in Sec. III either as a wormhole or particle. Our conclusion in Sec. IV completes the paper.

\section{ACTION AND FIELD EQUATIONS}

The $(2+1)$-dimensional action in the Einstein gravity coupled to a scalar field, without cosmological constant and self-interacting potential, is given by $(16 \pi G=c=1)$,

$$
S=\int d^{3} x \sqrt{-g}\left(R-\frac{\epsilon}{2}\left(\nabla \phi^{a}\right)^{2}\right)
$$

in which $\epsilon=+1 /-1$ corresponds to the normal/phantom scalar field where $\phi^{a}$ is the doublet scalar field with $a=1,2$. The standard form of the line element for a 
wormhole in circularly symmetric $(2+1)$-dimensional spacetime is given by

$$
d s^{2}=-e^{2 \Phi} d t^{2}+\frac{1}{1-\frac{b(r)}{r}} d r^{2}+r^{2} d \theta^{2} .
$$

Here $\Phi=\Phi(r)$ is the redshift function and $b(r)$ is the shape function satisfying the so-called flare-out conditions to which we shall refer in the sequel. Our doublet scalar field ansatz is given by

$$
\phi^{a}=\eta f(r) \frac{x^{a}}{r},
$$

where $x^{1}=r \cos \theta$ and $x^{2}=r \sin \theta, \eta$ is a coupling constant and $f(r)$ is a real function of $r$. This ansatz is well known from the particlelike global monopole solution in the gravity coupled field theory model [4]. It admits topological properties, and due to its angular dependence it exhibits nonisotropic properties in the radial plane. In particular, the asymptotic behaviors are comparable with those of cosmic strings which are known to possess deficit angles. Such a model gives rise to lumpy structures in cosmic formations and naturally modifies all tests of general relativity ranging from planetary motion to light bending. The reality of the model can only be tested by comparing geodesics of all kinds with the experimental data.

Considering the doublet field given in (3), one finds

$$
\left(\nabla \phi^{a}\right)^{2}=\eta^{2}\left(\left(1-\frac{b}{r}\right) f^{\prime 2}+\frac{f^{2}}{r^{2}}\right)
$$

such that after applying the variation of the action with respect to $f$, the field equation becomes

$$
f^{\prime \prime}+\left(\Phi^{\prime}+\frac{2 r-\left(b+r b^{\prime}\right)}{2 r(r-b)}\right) f^{\prime}-\frac{f}{r(r-b)}=0 .
$$

We note that a prime stands for the derivative with respect to $r$. Einstein's equations are given as

$$
G_{\mu}^{\nu}=T_{\mu}^{\nu}
$$

for

$$
T_{\mu}^{\nu}=\frac{\epsilon}{2}\left(\partial_{\mu} \phi^{a} \partial^{\nu} \phi^{a}-\frac{1}{2} \partial_{\rho} \phi^{a} \partial^{\rho} \phi^{a} \delta_{\mu}^{\nu}\right)
$$

The latter implies

$$
\begin{gathered}
T_{t}^{t}=-\epsilon \frac{\eta^{2}}{4}\left(\left(1-\frac{b}{r}\right) f^{\prime 2}+\frac{1}{r^{2}} f^{2}\right), \\
T_{r}^{r}=\epsilon \frac{\eta^{2}}{4}\left(\left(1-\frac{b}{r}\right) f^{\prime 2}-\frac{1}{r^{2}} f^{2}\right),
\end{gathered}
$$

and

$$
T_{\theta}^{\theta}=-T_{r}^{r}
$$

Accordingly, Einstein's equations read

$$
\begin{aligned}
\frac{b-r b^{\prime}}{2 r^{3}} & =-\epsilon \frac{\eta^{2}}{4}\left(\left(1-\frac{b}{r}\right) f^{\prime 2}+\frac{1}{r^{2}} f^{2}\right), \\
\frac{\left(r-b^{\prime}\right) \Phi^{\prime}}{r^{2}} & =\epsilon \frac{\eta^{2}}{4}\left(\left(1-\frac{b}{r}\right) f^{\prime 2}-\frac{1}{r^{2}} f^{2}\right),
\end{aligned}
$$

and

$$
\begin{aligned}
& \frac{2 r(r-b) \Phi^{\prime \prime}+2 \Phi^{\prime}\left(r(r-b) \Phi^{\prime}+\frac{1}{2}\left(b-r b^{\prime}\right)\right)}{2 r^{2}} \\
& \quad=-\epsilon \frac{\eta^{2}}{4}\left(\left(1-\frac{b}{r}\right) f^{\prime 2}-\frac{1}{r^{2}} f^{2}\right) .
\end{aligned}
$$

In the next section we shall find an exact solution for the four field equations given in (5), (11), (12), and (13).

\section{EXACT SOLUTIONS}

The field equations admit an exact solution for $\Phi=0$. The field equations, in this setting, become

$$
\begin{gathered}
f^{\prime \prime}+\left(\frac{2 r-\left(b+r b^{\prime}\right)}{2 r(r-b)}\right) f^{\prime}-\frac{f}{r(r-b)}=0, \\
\frac{b-r b^{\prime}}{2 r^{3}}=-\epsilon \frac{\eta^{2}}{4}\left(\left(1-\frac{b}{r}\right) f^{\prime 2}+\frac{1}{r^{2}} f^{2}\right),
\end{gathered}
$$

and

$$
\left(1-\frac{b}{r}\right) f^{\prime 2}-\frac{1}{r^{2}} f^{2}=0 .
$$

The last equation implies

$$
b=\left(r-\frac{1}{r} \frac{f^{2}}{f^{\prime 2}}\right),
$$

and upon substitution into (14), one finds that it is satisfied. Therefore, the only equation left becomes

$$
2 r f^{\prime 2}-2 f f^{\prime}-2 r f^{\prime \prime} f+\epsilon \eta^{2} r^{2} f f^{\prime 3}=0,
$$

which can be rewritten as

$$
\left(\frac{f}{r f^{\prime}}\right)^{\prime}=-\frac{1}{2} \epsilon \eta^{2} f f^{\prime} .
$$

An integration yields 


$$
\frac{f}{r f^{\prime}}=-\frac{\epsilon \eta^{2}}{4} f^{2}+C_{1},
$$

with the integration constant $C_{1}$. The resulting equation simply reads

$$
\frac{d r}{r}=\left(-\frac{\epsilon \eta^{2}}{4} f+\frac{C_{1}}{f}\right) d f
$$

which is integrable as

$$
\ln \left(\frac{r}{r_{0}}\right)=-\frac{\epsilon \eta^{2}}{8} f^{2}+C_{1} \ln f
$$

with $r_{0}$ another integration constant. Finally, $f$ is found to be

$$
f=\left(\frac{r}{r_{0}}\right)^{\xi} \exp \left[-\frac{1}{2} L W\left(-\frac{\epsilon \eta^{2} \xi}{4}\left(\frac{r}{r_{0}}\right)^{2 \xi}\right)\right]
$$

in which $\xi=\frac{1}{C_{1}}$ and $L W(x)$ is the Lambert-W function [14]. Using (23) we also find the exact form of $b(r)$ which is determined as

$$
b=r\left[1-\frac{\left(1+L W\left(-\frac{\epsilon \eta^{2} \xi}{4}\left(\frac{r}{r_{0}}\right)^{2 \xi}\right)\right)^{2}}{\xi^{2}}\right]
$$

The only nonzero component of the energy momentum tensor is $T_{0}^{0}=-\rho$ in which the energy density is given by

$$
\rho=-\frac{2}{r^{2} \xi} L W\left(-\frac{\epsilon \eta^{2} \xi}{4}\left(\frac{r}{r_{0}}\right)^{2 \xi}\right) .
$$

In these solutions there are four parameters: $\eta$ and $\epsilon$ from the action and $r_{0}$ and $\xi=\frac{1}{C_{1}}$ as integration constants. Setting $\eta=0$ directly yields $\phi^{a}=0$ and $b(r)=\frac{1}{\xi^{2}}$ which corresponds to the flat spacetime. Due to the quadratic form of $\eta^{2}$, both in the action and in the solution, $\eta \lessgtr 0$ have similar contribution. Also, $r_{0}$ is a scale factor with dimension as $r$ and, therefore, we restrict $r_{0}>0$. Unlike $\eta$, the sign of the other two parameters brings different features for the general solutions. Here we study each case separately.

$$
\text { A. } \epsilon=1, \xi>0
$$

The first setup corresponds to $\epsilon=1, \xi>0$. In this setting, $f(r)$ is defined for $r<r_{c}=(2 / \eta \sqrt{\xi e})^{1 / \xi} r_{0}$ and, therefore, the solution is bounded from above, and we shall call it a particle model. In this confined model, the particle is supported by normal matter with $\rho>0$.

$$
\text { B. } \epsilon=1, \xi<0
$$

The second setup for the two free parameters is considered as $\epsilon=1$ and $\xi<0$. In this case the line element can be written as

$$
d s^{2}=-d t^{2}+\frac{1}{B(r)} d r^{2}+r^{2} d \theta^{2},
$$

where

$$
B(r)=\frac{1}{\xi^{2}}\left(1+L W\left(-\frac{\eta^{2} \xi}{4}\left(\frac{r}{r_{0}}\right)^{2 \xi}\right)\right)^{2}
$$

which is positive for $r>0$. For $r=0$, there exists a singularity while for large $r, B(r)$ asymptotes to $\frac{1}{\xi^{2}}$. Therefore, without loss of generality, one may set $\xi=-1$. (We note that unlike the $(3+1)$-dimensional spacetime where

$$
d s^{2}=-d t^{2}+\xi^{2} d r^{2}+r^{2}\left(d \theta^{2}+\sin ^{2} \theta d \phi^{2}\right)
$$

is flat only if $\xi^{2}=1$, in $2+1$ dimensions for any value of $\xi \neq 0$, the spacetime is flat.) In Fig. 1 we plot $B(r)$ in terms of $r$ for various values for $\eta$. The solution is supported by the normal matter of the doublet scalar field which is naked singular at $r=0$ and asymptotically flat. We observe from this figure that the larger value of $\eta^{2}$ makes the spacetime more deviated from the flat spacetime corresponding to $\eta^{2}=0$. Therefore, the larger the $\eta^{2}$, the stronger the doublet scalar fields, which results in stronger curvature. Having $\epsilon=1$ in the action makes the scalar fields physical and also $\xi<0$ makes the energy density $\rho>0$. Therefore, this solution represents a naked

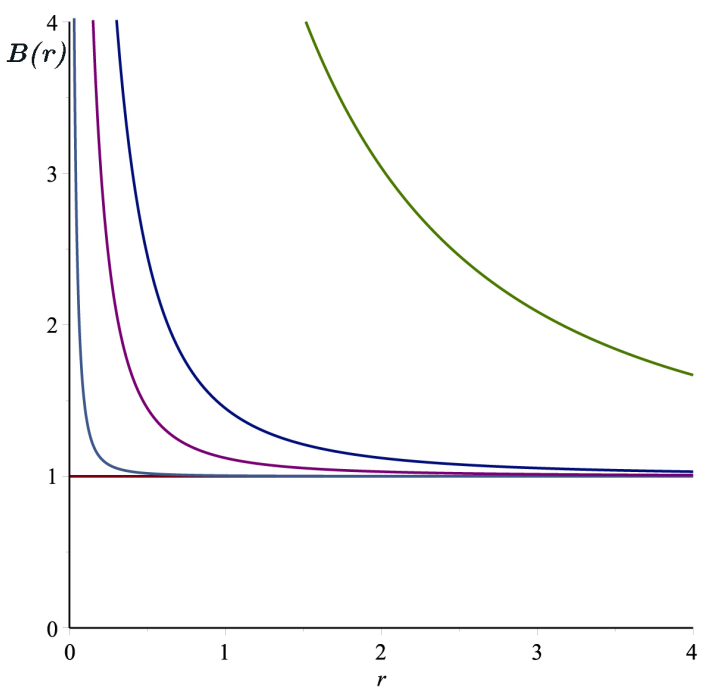

FIG. 1 (color online). $B(r)$ versus $r$ [Eq. (27)] for various values of $\eta=5.0,1.0,0.5,0.1$, and 0.0 from top to bottom, respectively, with $\xi=-1, \epsilon=1, r_{0}=1$. 
singular solution supported by a normal doublet of the scalar field which is asymptotically flat. This solution can also be interpreted as a particle model constructed from the doublet of the scalar fields. To complete this part, we add that the field function $f(r)$ is well defined for $r>0$ and its asymptotic behaviors are

$$
\lim _{r \rightarrow 0} f(r)=\infty
$$

and

$$
\lim _{r \rightarrow \infty} f(r)=0
$$

It is observed that the source of the field looks to be diverging at $r=0$, where the spacetime is curved maximally and is singular.

$$
\text { C. } \epsilon=-1, \xi>0
$$

In this setting for $\epsilon=-1$ and $\xi>0$ the solution is exotic, supported by negative energy density. The field function $f(r)$ is well defined for $r>0$, and while at $r=0$ it vanishes, at large $r$ it diverges.

In Fig. 2 we plot $B(r)$ versus $r$ for different values of $\eta$. The solution is supported by the exotic matter/phantom doublet of scalar fields, which is flat near $r=0$ and nonasymptotically flat for $r \rightarrow \infty$. The larger value of $\eta^{2}$ makes the spacetime more deviated from the flat spacetime with $\eta^{2}=0$. Note that the asymptotic behaviors of the solution at small $r$ and large $r$ in the present case look to be the opposite of the previous case. The two cases are still different solutions and by a change of variable, for instance $r \rightarrow \frac{1}{r}$, it is not possible to obtain one from the other.

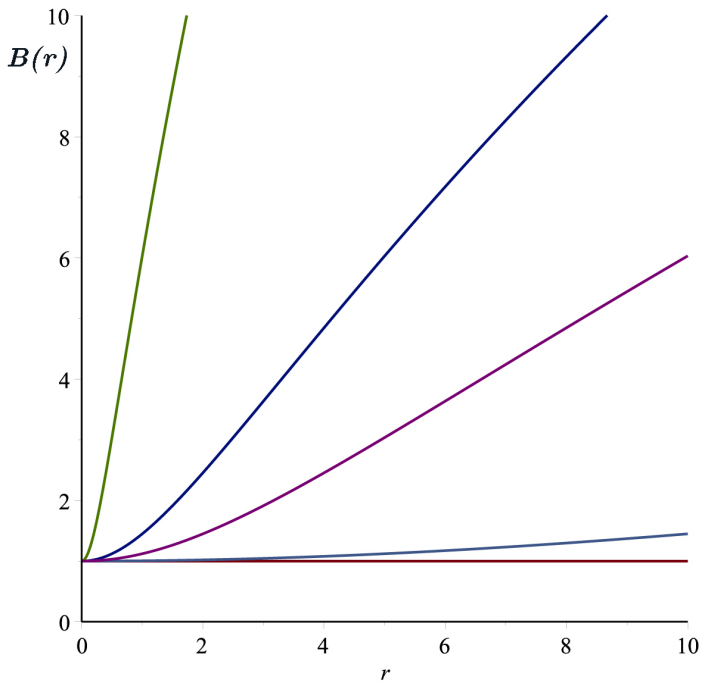

FIG. 2 (color online). $B(r)$ versus $r$ [Eq. (27)] for various values of $\eta=5.0,1.0,0.5,0.1$, and 0.0 from top to bottom, respectively, with $\xi=1, \epsilon=-1, r_{0}=1$.

\section{Wormhole solution for $\epsilon=-1, \xi<0$}

Our last general setting addresses the most interesting case, where $\epsilon=-1, \xi<0$ and the solution represents a wormhole with a throat located at

$$
b_{0}=r_{0}\left(\frac{e \eta^{2}|\xi|}{4}\right)^{\frac{1}{2|\xi|}}
$$

in which $e$ stands for the natural base of logarithm. The wormhole is asymptotically flat with

$$
\lim _{r \rightarrow \infty} B(r)=\frac{1}{\xi^{2}},
$$

where we shall choose $\xi=-1$. Both $b(r)$ and $f(r)$ are positively defined for $r>b_{0}$, and $b(r)$ satisfies the flareout conditions; i.e., (i) $b\left(b_{0}\right)=b_{0}$ and (ii) for $r>b_{0}, r b^{\prime}<b$ such that the field function smoothly vanishes at infinity from its maximum value $\frac{\sqrt{2}}{|\eta|}$ at the throat. In terms of the throat radius, one may write

$$
\begin{aligned}
& f=\frac{2\left(\frac{b_{0}}{r}\right)^{|\xi|}}{\eta \sqrt{e|\xi|}} \exp \left[-\frac{1}{2} L W\left(\frac{-1}{e}\left(\frac{b_{0}}{r}\right)^{2|\xi|}\right)\right], \\
& b=r\left(1-\frac{1}{\xi^{2}}\left[1+L W\left(\frac{-1}{e}\left(\frac{b_{0}}{r}\right)^{2|\xi|}\right)\right]^{2}\right)
\end{aligned}
$$

with the scalar invariants given by

$$
\begin{gathered}
K=R_{\mu \nu \alpha \beta} R^{\mu \nu \alpha \beta}=\frac{16 L W\left(\frac{-1}{e}\left(\frac{b_{0}}{r}\right)^{2|\xi|}\right)^{2}}{r^{4} \xi^{2}}, \\
R_{\mu \nu} R^{\mu \nu}=\frac{8 L W\left(\frac{-1}{e}\left(\frac{b_{0}}{r}\right)^{2|\xi|}\right)^{2}}{r^{4} \xi^{2}},
\end{gathered}
$$

and

$$
R=R_{\mu}^{\mu}=\frac{4 L W\left(\frac{-1}{e}\left(\frac{b_{0}}{r}\right)^{2|\xi|}\right)}{r^{2}|\xi|} .
$$

The only nonzero component of the energy momentum tensor is the $t t$ component which is given by

$$
T_{t}^{t}=-\rho=-\frac{2 L W\left(\frac{-1}{e}\left(\frac{b_{0}}{r}\right)^{2|\xi|}\right)}{r^{2}|\xi|} .
$$

Let us add that on the range of $r$, i.e., $r \geq b_{0}$, all of the quantities given above are finite and they vanish asymptotically. In addition, one finds 


$$
\begin{array}{cc}
\lim _{r \rightarrow b_{0}^{+}} f=\frac{2}{\eta \sqrt{|\xi|}}, & \lim _{r \rightarrow b_{0}^{+}} b=b_{0}, \\
\lim _{r \rightarrow b_{0}^{+}} \rho=-\frac{2}{b_{0}^{2} \sqrt{|\xi|}}, & \lim _{r \rightarrow b_{0}^{+}} K=\frac{16}{b_{0}^{4} \xi^{2}},
\end{array}
$$

and

$$
\lim _{r \rightarrow b_{0}^{+}} R_{\mu \nu} R^{\mu \nu}=\frac{8}{b_{0}^{4} \xi^{2}}, \quad \lim _{r \rightarrow b_{0}^{+}} R=-\frac{4}{b_{0}^{2}|\xi|} .
$$

In Fig. 3(a) we plot the scalars given above to show that they are finite everywhere, and in Fig. 3(b) the curve of
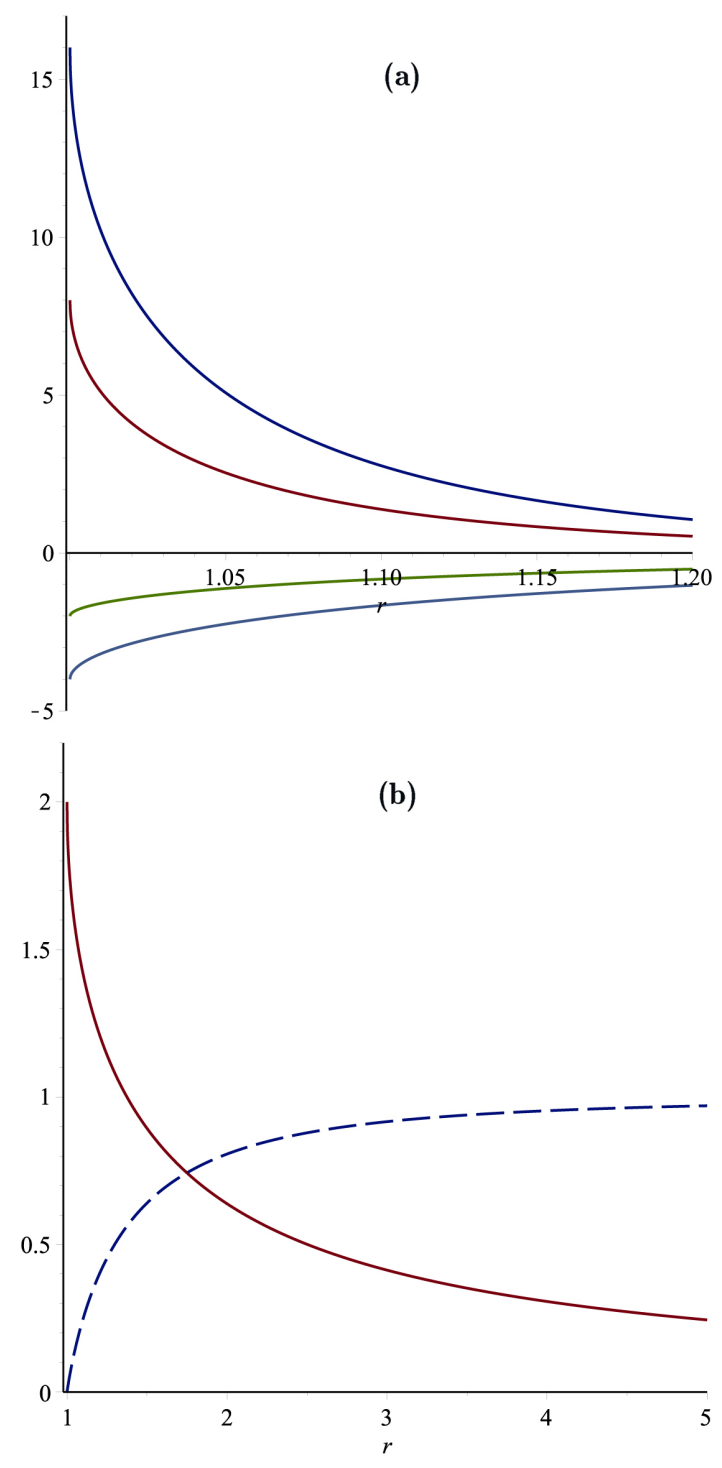

FIG. 3 (color online). (a) From top to bottom [Eqs. (35)-(38)]; $K, R_{\mu \nu} R^{\mu \nu}, \rho$, and $R$ versus $r>b_{0}$ (b) $B(r)$ (dashed) and $\eta f(r)$ (solid) in terms of $r$ for $r>b_{0}$. For both we set $b_{0}=1, \epsilon=-1$, and $\xi=-1$.

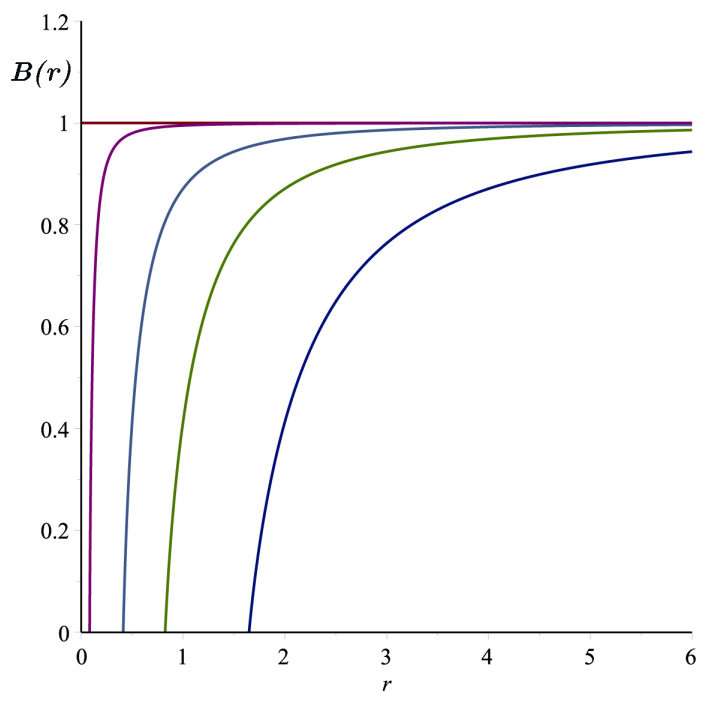

FIG. 4 (color online). $\quad B(r)$ versus $r$ from Eq. (27) for various values of $\eta=5.0,1.0,0.5,0.1$, and 0.0 from bottom to top, respectively, with $\xi=-1, \epsilon=-1, r_{0}=1$.

energy density $\rho$ together with the corresponding metric function $B(r)$ are displayed. The energy density is negative everywhere but finite, indicating the wormhole is supported by exotic matter [10]. In Fig. 4 we plot $B(r)$ versus $r$ for different values of $\eta$ with fixed values for $\xi=-1$ and $r_{0}=1$ (note that with $r_{0}=1$ and different values for $\eta$, the throat $b_{0}$ is not fixed). The magnitude of $\eta$ plays a critical role to form the throat of the wormhole such that the larger value for $\eta$ implies a larger size of the throat.

\section{E. $\boldsymbol{\xi}=\mathbf{0}$ and $\boldsymbol{\xi}=\infty$}

Among the possible values for $\xi$ the case with $\xi=0$ corresponds to $f=1$ and consequently to the flat space solution. In contrast to that, when $\xi \rightarrow \infty$, the solution becomes (this can be seen from (22) when $C_{1}=0$ )

$$
f^{2}=-\frac{8}{\epsilon \eta^{2}} \ln \left(\frac{r}{r_{0}}\right)
$$

so that

$$
b=r\left(1-4\left(\ln \frac{r}{r_{0}}\right)^{2}\right)
$$

and

$$
d s^{2}=-d t^{2}+\frac{d r^{2}}{\left(2 \ln \frac{r}{r_{0}}\right)^{2}}+r^{2} d \theta^{2} .
$$

This line element has the following scalar invariants:

$$
R=R_{\mu}^{\mu}=-\frac{8 \ln \frac{r}{r_{0}}}{r^{2}}
$$




$$
K=R_{\mu \nu \alpha \beta} R^{\mu \nu \alpha \beta}=\frac{64 \ln ^{2} \frac{r}{r_{0}}}{r^{4}},
$$

and

$$
R_{\mu \nu} R^{\mu \nu}=\frac{32 \ln ^{2} \frac{r}{r_{0}}}{r^{4}}
$$

It is seen clearly that $r=0$ is a spacetime singularity while at $r=r_{0}$ it is regular. This metric cannot be interpreted as a wormhole since from (43) as $r>r_{0}$ the sign of $b(r)$ turns negative which is in contrast to the definition of a wormhole. The only nonzero component of the energymomentum tensor is given by

$$
T_{t}^{t}=\frac{4 \ln \frac{r}{r_{0}}}{r^{2}},
$$

with a divergent energy density at the origin given by

$$
\rho=-T_{t}^{t}
$$

\section{CONCLUSION}

For a number of reasons in recent decades the lower-/ higher-dimensional curved spacetimes received much attention. Our aim in this paper was to consider a doublet of nonisotropic scalar fields $\phi^{a}(r, \theta)$ transforming under the group $O(2)$. We present parametric solutions for such a system to determine the underlying $(2+1)$-dimensional spacetime. Our solution involves the restrictive condition of the vanishing redshift function. Making $g_{t t}=-1$ leaves us with a single metric function $g_{r r}=\frac{1}{B(r)}$ besides $\phi^{a}(r, \theta)$. Once the redshift function vanishes, our solution loses its chance to represent a black hole. However, the wormhole and particle interpretations are admissible, and as a matter of fact, this summarizes the contribution made in this paper. Our only metric function as well as the doublet scalar functions are expressed in terms of a Lambert function which is tabulated extensively in the literature. The source supporting our wormhole turns out to be exotic, which persists in being a deep-rooted problem in general. We wish to remark finally that in order to overcome this problem of exoticity, we recently proposed a resolution, which is to change the circular topological character of the throat [15].
[1] C. Barcelo and M. Visser, Classical Quantum Gravity 17, 3843 (2000); S.-W. Kim and S. P. Kim, Phys. Rev. D 58, 087703 (1998); O. Hauser, R. Ibadov, B. Kleihaus, and J. Kunz, Phys. Rev. D 89, 064010 (2014); A. Anabalón, D. Astefanesei, and R. Mann, J. High Energy Phys. 10 (2013) 184; T. Torii and H.-a. Shinkai, Phys. Rev. D 88, 064027 (2013).

[2] M. Gell-Mann and M. Lévy, Il Nuovo Cimento 16, 705 (1960).

[3] P. W. Higgs, Phys. Rev. Lett. 13, 508 (1964).

[4] M. Barriola and A. Vilenkin, Phys. Rev. Lett. 63, 341 (1989).

[5] G. P. Perry and R. B. Mann, Gen. Relativ. Gravit. 24, 305 (1992); S. W. Kim, H. J. Lee, S. K. Kim, and J. Yang, Phys. Lett. A 183, 359 (1993); M. S. R. Delgaty and R. B. Mann, Int. J. Mod. Phys. D 04, 231 (1995); W. T. Kim, J. J. Oh, and M. S. Yoon, Phys. Rev. D 70, 044006 (2004); F. Rahaman, A. Banerjee, and I. Radinschi, Int. J. Theor. Phys. 51, 1680 (2012); A. Banerjee, Int. J. Theor. Phys. 52, 2943 (2013); C. Bejarano, E. F. Eiroa, and C. Simeone, Eur. Phys. J. C 74, 3015 (2014).

[6] S. H. Mazharimousavi and M. Halilsoy, arXiv:1408.3008.
[7] A. Einstein and N. Rosen, Phys. Rev. 48, 73 (1935).

[8] M. S. Morris and K. S. Thorne, Am. J. Phys. 56, 395 (1988).

[9] K. S. Virbhadra, Pramana 44, 317 (1995).

[10] M. Visser, S. Kar, and N. Dadhich, Phys. Rev. Lett. 90, 201102 (2003); S. V. Bolokhov, K. A. Bronnikov, and M. V. Skvortsova, Classical Quantum Gravity 29, 245006 (2012).

[11] K. A. Bronnikov, S. V. Chervon, and S. V. Sushkov, Gravitation Cosmol. 15, 241 (2009).

[12] M. Jamil and M. U. Farooq, Int. J. Theor. Phys. 49, 835 (2010).

[13] M. Bañados, C. Teitelboim, and J. Zanelli, Phys. Rev. Lett. 69, 1849 (1992); M. Bañados, M. Henneaux, C. Teitelboim, and J. Zanelli, Phys. Rev. D 48, 1506 (1993); C. Martinez, C. Teitelboim, and J. Zanelli, Phys. Rev. D 61, 104013 (2000); S. Carlip, Quantum Gravity in 2+1-Dimensions (Cambridge University Press, Cambridge, England, 1998).

[14] R. M. CorlessG. H. Gonnet, D. E. G. Hare, D. J. Jeffrey, and D. E. Knuth, Adv. Comput. Math. 5, 329 (1996).

[15] S. H. Mazharimousavi and M. Halilsoy, Eur. Phys. J. C 75, $81(2015)$. 